\title{
Clinical and computed tomographic (CT) images characteristics in the patients with COVID-19 infection: What should radiologists need to know?
}

\author{
Pinggui Lei ${ }^{\mathrm{a}, 1, *}$, Zhaoshu Huang ${ }^{\mathrm{a}, 1}$, Guoli Liu ${ }^{\mathrm{a}, 1}$, Pingxian Wang ${ }^{\mathrm{b}}$, Wen Song ${ }^{\mathrm{a}}$, \\ Jujiang $\mathrm{Mao}^{\mathrm{a}, *}$, Guiquan Shen ${ }^{\mathrm{a}}$, Shi Zhou ${ }^{\mathrm{a}}$, Wei Qian ${ }^{\mathrm{c}}$ and Jun Jiao ${ }^{\mathrm{a}}$ \\ ${ }^{a}$ Department of Radiology, the Affiliated Hospital of Guizhou Medical University. Guiyang, China \\ ${ }^{\mathrm{b}}$ Department of Medical Insurance, the Affiliated Hospital of Guizhou Medical University, \\ Guiyang, China \\ ${ }^{\mathrm{c}}$ Department of Electrical and Computer Engineering, College of Engineering, \\ University of Texas, El Paso, TX, USA
}

Received 26 February 2020

Revised 5 March 2020

Accepted 9 March 2020

\begin{abstract}
.
OBJECTIVE: To evaluate the clinical and computed tomographic (CT) features in the patients with COVID-19 pneumonia confirmed by the real-time reverse transcriptase polymerase chain reaction (rRT-PCR) amplification of the viral DNA from a sputum sample.

MATERIAL AND METHODS: Clinical information and CT findings of a total of 14 patients with COVID-19 infection (age range, 12-83 years; females 6) were analyzed retrospectively. The clinical information includes the history of exposure, laboratory results, and the symptoms (such as fever, cough, headache, etc.); CT findings of chest include the extension and distribution of lesion, the ground-glass opacity (GGO), consolidation, bronchovascular enlarged, irregular linear appearances, pleural effusion, and lymphadenopathy.

RESULTS: Eight patients had the exposure history for recent travel to Wuhan of Hubei province (8/14, 57\%), 6 had the exposure to patients with COVID-19 infection. Significant statistical differences were observed in lymphocyte percentage decreased and C-reactive protein elevated $(p=0.015)$. Seven patients had fever, 7 had cough, 2 had headache, 3 had fatigue, 1 had body soreness, 3 had diarrhea, and 2 had no obvious symptoms. In chest CT examination, 10 patients were positive $(10 / 14,71.43 \%)$. Among these patients, 9 had lesions involving both lungs (9/10, 90\%), 8 had lesions involving 4 to 5 lobes $(8 / 10,80 \%)$. Most of lesions were distributed peripherally and the most significant lesions were observed in the right lower lobe in 9 patients $(9 / 10,90 \%)$. Nodules were observed in 5 patients $(5 / 10,50 \%)$; GGO, consolidation, and bronchovascular enlarged were shown in 9 patients $(9 / 10,90 \%)$; irregular linear appearances were revealed in 7 patients $(7 / 10,70 \%)$; and pleural effusions were exhibited in 2 patients $(2 / 10,20 \%)$. Last, no patients showed lymphadenopathy.

CONCLUSION: There were some typical CT features for diagnosis of COVID-19 pneumonia. The radiologists should know these CT findings and clinical information, which could help for accurate analysis in the patients with 2019 novel coronavirus infection.
\end{abstract}

Keywords: Novel coronavirus, virus pneumonia, COVID-19 infection, computed tomographic findings

\footnotetext{
${ }^{1}$ These authors are equal contributors.

${ }^{*}$ Corresponding author: Pinggui Lei and Jujiang Mao, Department of Radiology, the Affiliated Hospital of Guizhou Medical University. Guiyang of Guizhou 550004, China. (Pinggui Lei), E-mails: pingguilei@ foxmail.com; (Jujiang Mao), 51502946@qq.com.
} 


\section{Introduction}

Current novel outbreak of coronavirus disease (COVID-19) was named by World Health Organization (WHO), and first reported in December 2019 in Wuhan, the capital of Hubei province, in the center area of China. The early transmission of COVID-19 has been confirmed human-to-human [1]. As we all know, the history of virus infection to human beings for many times, such as the Ebola infection to human, H1N1 etc. [2, 3]. Until Feb. 25, 2020, the World Health Organization (WHO) had received 80,239 reports (908 new) of confirmed cases and 2,700 deaths (82 new) all around the world, and more than 30 countries are affected. In addition, outside China, the COVID-19 was also confirmed at the same time in Korea, Japan, Singapore, Italy, American, etc. [4]. Currently, Chest X-ray and CT scan were performed as the image modality methods for detecting the COVID-19 pneumonia [5, 6]. However, chest X-ray could not provide the cross-sectional images information, the muscle, bone, and other structure can block valuable diagnostic data to detect the early features of COVID-19 pneumonia. With the development of CT examination utilized in clinical practice, the lesions have been discovered in the lung increased. In the previous coronavirus outbreaks, CT is more sensitive likely to detect early and/or mild lesions [7]. The recently literature reported that chest CT can be applied to screening in the patients with suspected COVID-19 pneumonia when the rRT-PCR testing is negative [8]. Therefore, chest CT examination performed in the patients is a feasible solution to make mutual confirmation between nuclear acid results and radiological features.

Recently, fever, cough, headache, fatigue, nausea, and vomit could be valuable clinical information for diagnosis of COVID-19 pneumonia [1, 9, 10]. Besides the clinical information, the radiologists should recognize and understand the CT features of COVID-19 pneumonia, literature revealed that bilateral consolidation pulmonary opacity and GGO in the pulmonary parenchyma were the typical CT findings [11-13]. However, a little know about this novel coronavirus, the details and evolution of CT features for COVID-19 infection were still confused and needed further study and discussion. What's more, we should understand the laboratory results, positive CT feature and the negative CT features. In our study, the CT features of GGO and consolidation, the distribution of lesions, involvement of lobes, bronchovascular enlarged, irregular linear appearances, pleural effusion, lymphadenopathy, and laboratory results were evaluated in the patients with COVID-19 infection, which may help radiologists to make diagnosis in the patients with suspected COVID-19 infection for their isolation and treatment.

As the transmission of human-to-human for the COVID-19 pneumonia, rapid recognition and diagnosis of this disease is essential importance for the patient to avoid containment of the health individuals or of their family. In this study, we reported the main clinical manifestation and key CT findings in a serial of 14 patients with COVID-19 infection who confirmed by rRT-PCR amplification of the viral DNA from a sputum sample.

\section{Materials and methods}

This study was approved by the Ethics of committees in our hospital, the informed consent was waived for this retrospective study.

\subsection{Patients}

Clinical manifestation, laboratory examination, and chest CT images of 14 consecutive patients with COVID-19 pneumonia were retrospectively analyzed from January 16, 2020 to February 22, 2020 in our hospital. The patients' history of exposure and clinical manifestation (fever, cough, headache, fatigue, nausea, vomit, body soreness, and no significant symptoms) were collected in Hospital Information System (HIS) of our hospital. All of 14 patients were performed pre-contrast chest CT examination 
before treatment. The days were also recorded for the patients with COVID-19 pneumonia from the onset of chief symptom to the first CT scan.

\subsection{Chest CT protocols}

The patients were mainly performed on 128-slice MSCT scanning (SOMATOM Definition AS+, Siemens, Germany) or 16-slice MSCT scanning (Aquilion16, Toshiba Medical, Nasu, Japan). For 128-slice MSCT scanning, the scan parameters were as follows: Tube voltage $120 \mathrm{kV}$, tube current $150 \mathrm{mAs}$, pitch 1.2, collimation $128 \times 0.6 \mathrm{~mm}$, slice thickness 1 or $5 \mathrm{~mm}$, FOV $360 \mathrm{~mm}$. For 16-slice MSCT scanning, the scan parameters were as follows: Tube voltage $120 \mathrm{kV}$, tube current $150 \mathrm{mAs}$, pitch 0.8 , collimation $128 \times 0.6 \mathrm{~mm}$, slice thickness 1 or $5 \mathrm{~mm}$, FOV $360 \mathrm{~mm}$.

\subsection{Diagnostic criteria for the patients with COVID-19 pneumonia}

According to COVID-19 pneumonia diagnosis and treatment plan promulgated by National Health Commission of the People's Republic of China. Two steps in conclusion for diagnosis of COVID-19 are as following:

The First Step - Diagnosis of the suspected cases: For this purpose, comprehensive analysis for epidemiological exposure history and clinical manifestations in diagnosis of COVID infection are performed. First, 4 epidemiological history are examined including (1) a history of travel or residence in Wuhan city and surrounding areas, or other communities with case reports within 14 days before the onset; (2) a history of contact with COVID-19 patients (nucleic acid test positive) within 14 days prior to onset; (3) a history of exposure to the patients with fever or respiratory symptoms from the city of Wuhan and its surrounding areas, or from the community with reported cases within 14 days before the onset; and (4) cluster onset. Next, 3 clinical manifestations are checked including (1) fever and/or respiratory symptoms; (2) with the COVID-19 imaging features; and (3) at the early stage, the total number of white blood cells was normal or decreased, and the count of lymphocytes decreased. In current clinical practice, patients with one of the following two conditions are considered suspected cases namely, (1) the patients who have any one of four aspects in the epidemiological history and any two of three aspects in the clinical manifestations, and (2) the patients who have no clear epidemiological history, but have three aspects of the clinical manifestations.

The Second Step - Confirmation of COVID-19 infected cases: The suspected cases with one of the following pathogenic evidences are confirmed as COVID-19 infected cases namely, (1) RT-PCR detection of new coronavirus nucleic acid is positive; and (2) the novel coronavirus is highly homologous to the COVID-19.

\subsection{Evaluation of chest CT findings}

When chest CT examination was over, all the CT images dataset were reconstructed at the workstation with filtered back projection algorithm based on its original dataset. CT images with $1 \mathrm{~mm}$ thickness and no intersection gap were generated for chest CT datasets. Multiplanar reformation (MPR) images (including axial, sagittal, coronal image) were also generated, and these images were subsequently loaded into picture archiving and communication system (PACS) workstation (Syngo.plaza, Siemens) for interpretation in consensus by two experienced radiologists (P.G.L. and J.J.M) with approximately 6 years and 20 years, respectively.

At first, the overall image quality of the patient with COVID-19 infection was evaluated: the overall image quality degree was scored as poor (score 1), suboptimal (score 2), diagnostic (score 3), superior (score 4), excellent (score 5). The image quality score $\geq 3$ was considered as satisfaction for evaluation in clinical practice. 
The CT characteristics were evaluated as following: (1) the distribution of lesions: a. periphery, b. centrality (around bronchovascular), c. both; (2) involvement of lobes: a. one lobe, b. two lobes, c. three lobes, d. four lobes, e. five lobes; (3) presence of nodular; (4) presence of GGO; (5) presence of bronchovascular enlarged; (6) presence of irregular linear appearances; (7) presence of consolidation pulmonary opacity; (8) presence of pleural effusion; and (9) presence of lymphadenopathy. Last, the involvement of each of five lobes was also evaluated as following: none ( $0 \%$ involvement), mild $(1 \%-33 \%)$, moderate $(34 \%-66 \%)$, severe $(67-100 \%)$.

\subsection{Statistical analysis}

Statistical analysis was performed using SPSS software (version 23.0, SPSS Inc., Chicago, IL). Two groups of laboratory examination were classified according to the radiologic features of positive or negative chest CT results in the patients with COVID-19 infection. Fisher's exact test was used to compare laboratory indexes. $P<0.05$ was considered statistically significant difference.

\section{Results}

\subsection{Characteristics of patients with COVID-19 infection}

A total of 14 patients (males 8, females 6) with COVID-19 infection in this study, the average age 47 years, the standard deviation 19 years, age range from 12 years to 83 years. The patients' history of exposure for recent travel to Wuhan of Hubei province is $(8 / 14,57 \%)$, for exposure to patients with COVID-19 infection is $(6 / 14,43 \%)$, for unknown exposure is $(0 / 14,0 \%)$. As for the clinical manifestation: fever $(7 / 14,50.00 \%)$, cough $(7 / 14,50 \%)$, headache $(2 / 14,14 \%)$, fatigue $(3 / 14,21 \%)$, nausea $(0 / 14,0 \%)$, vomit $(0 / 14,0 \%)$, body soreness $(1 / 14,7 \%)$, diarrhea $(3 / 14,21 \%)$, and no significant symptoms $(2 / 14,14 \%)$. Of 14 patients with COVID-19 infection, 4 patients were presence of normal chest CT images $(4 / 14,29 \%)$, and the CT findings of other 10 patients were abnormal inordinately. The days recorded for the patients with COVID-19 pneumonia from the onset of chief symptom to the first CT scan were shown in Table 1. The Laboratory examination results were summarized in the TABLE 2. Significant statistical differences were observed in lymphocyte percentage decreased and C-reactive protein elevated (all $P=0.015$ ), and there were no significant statistical differences in the other laboratory examination results between positive and negative chest $\mathrm{CT}$ results in the patients with COVID-19 infection.

\subsection{Evaluation of chest CT features}

For subjective assessment of image quality in the patients with COVID-19 infection, the overall image quality was satisfactory for evaluating the CT features. Of 14 patients, 13 patients had an excellent score of image quality, one patient had superior score because of minimal movement artifact.

The CT features about the distribution of lesions as following: $(6 / 10,60 \%)$ cases periphery, $(0 / 10$, $0 \%$ ) cases centrality, $(4 / 10,40 \%)$ cases both; Involvement of lobes: $(1 / 10,10 \%)$ case involvement one lobe, $(1 / 10,10 \%)$ case involvement two lobe, $(1 / 10,10 \%)$ case involvement three lobes, $(3 / 10,30 \%)$ cases involvement four lobes, $(5 / 10,50 \%)$ cases involvement five lobes; Presence of nodular was up to $(5 / 10,50 \%)$; Presence of GGO was up to $(9 / 10,90 \%)$; Presence of bronchovascular enlarged was up to (9/10, 90\%); Presence of irregular linear appearances was up to (7/10, 70\%); Presence of consolidation pulmonary opacity was up to $(9 / 10,90 \%)$; Presence of pleural effusion was up to $(2 / 10,20 \%)$; There was no case with presence of lymphadenopathy $(0 / 10,0 \%)$. Several CT features were observed in $(9 / 10,90 \%)$ cases. (Please refer to Table 3) 
Table 1

The Patients' Characteristics

\begin{tabular}{|c|c|c|c|c|c|}
\hline $\begin{array}{l}\text { Patients } \\
\text { (No.) }\end{array}$ & Sex & $\begin{array}{c}\text { Age } \\
\text { (years) }\end{array}$ & Chief symptom & $\begin{array}{l}\text { Onset of chief } \\
\text { symptom to the first } \\
\text { CT scan (day }[\mathrm{s}] \text { ) }\end{array}$ & $\begin{array}{c}\text { Chest CT } \\
\text { examination }\end{array}$ \\
\hline
\end{tabular}

\begin{tabular}{|c|c|c|c|c|c|}
\hline 1 & male & 51 & body soreness, headache & 7 & + \\
\hline 2 & female & 61 & cough, diarrhea & 1 & + \\
\hline 3 & female & 53 & cough, no fever & 2 & + \\
\hline 4 & female & 30 & cough, fever & 2 & - \\
\hline 5 & male & 30 & diarrhea, fever & 5 & - \\
\hline 6 & male & 12 & no significant syptoms & travel to Wuhan 15 days ago & - \\
\hline 7 & male & 83 & fever, fatigue & 1 & + \\
\hline 8 & female & 50 & headache, fatigue, diarrhea & 3 & + \\
\hline 9 & male & 33 & fever, cough & 3 & + \\
\hline 10 & male & 64 & fatigue & 3 & + \\
\hline 11 & male & 47 & fever, cough & 1 & + \\
\hline 12 & female & 25 & fever, cough & 7 & - \\
\hline 13 & male & 49 & cough & 4 & + \\
\hline 14 & female & 63 & no significant syptoms & $\begin{array}{l}\text { exposure to COVID-19 } \\
\text { infection for } 7 \text { days }\end{array}$ & + \\
\hline
\end{tabular}

Note: “+” positive, “-” negative.

As for involvement of lobes, involvement of right upper lobe was up to (7/14, 50\%): mild involvement was $(4 / 14,28.57 \%)$, moderate involvement was $(1 / 14,7.14 \%)$, sever involvement was $(2 / 14,14.28 \%)$; Involvement of right middle lobe was up to $(6 / 14,42.86 \%)$ : mild was $(4 / 14,28.57 \%)$, moderate was $(1 / 14,7.14 \%)$, sever was $(1 / 14,7.14 \%)$; Involvement of right lower lobe was up to $(10 / 14,71.43 \%)$ : mild was $(6 / 14,42.86 \%)$, moderate was $(1 / 14,7.14 \%)$, sever was $(3 / 14,21.43 \%)$; Involvement of left upper lobe was up to $(8 / 14,57.14 \%)$ : mild was $(7 / 14,50.00 \%)$, sever was $(1 / 14,7.14 \%)$; Involvement of left lower lobe was up to $(9 / 14,64.29 \%)$ : mild was $(6 / 14,42.86 \%)$, moderate was $(1 / 14,7.14 \%)$, sever was $(2 / 14,14.28 \%)$.

\section{Discussion}

In this study, the results we got suggested that fever and cough were the main clinical manifestations in the patients with COVID-19 infection; Headache, diarrhea, and body soreness were relatively rare. Especially, two patients with COVID-19 infection were without any significant symptoms. As for the chest CT findings, most of lesions distributed periphery or peribronchovascular in the lung parenchymal. GGO, bronchovascular enlarged, and consolidation were the common radiological characteristics in the chest CT examination. Therefore, knowing these features is essential, because early detection of the lesions in the patients with suspected COVID-19 and precise diagnosis could be helpful to patients' isolation and treatment (FIGURE 1), and to avoid containment of the health individuals or their families.

All of 14 patients had the exposure history of recently travel to Wuhan (8/14) or exposure to the patients with COVID-19 infection (6/14). Fever, cough, diarrhea, sore throat, nausea and vomit, fatigue, and no significant symptoms, etc. were observed in the patients with COVID-19 infection [13-15]. In our study, the main clinical manifestation was fever, and cough. Diarrhea as the chief symptom was also discovered in 3 cases. However, only two patients without any significant symptoms, negative CT 


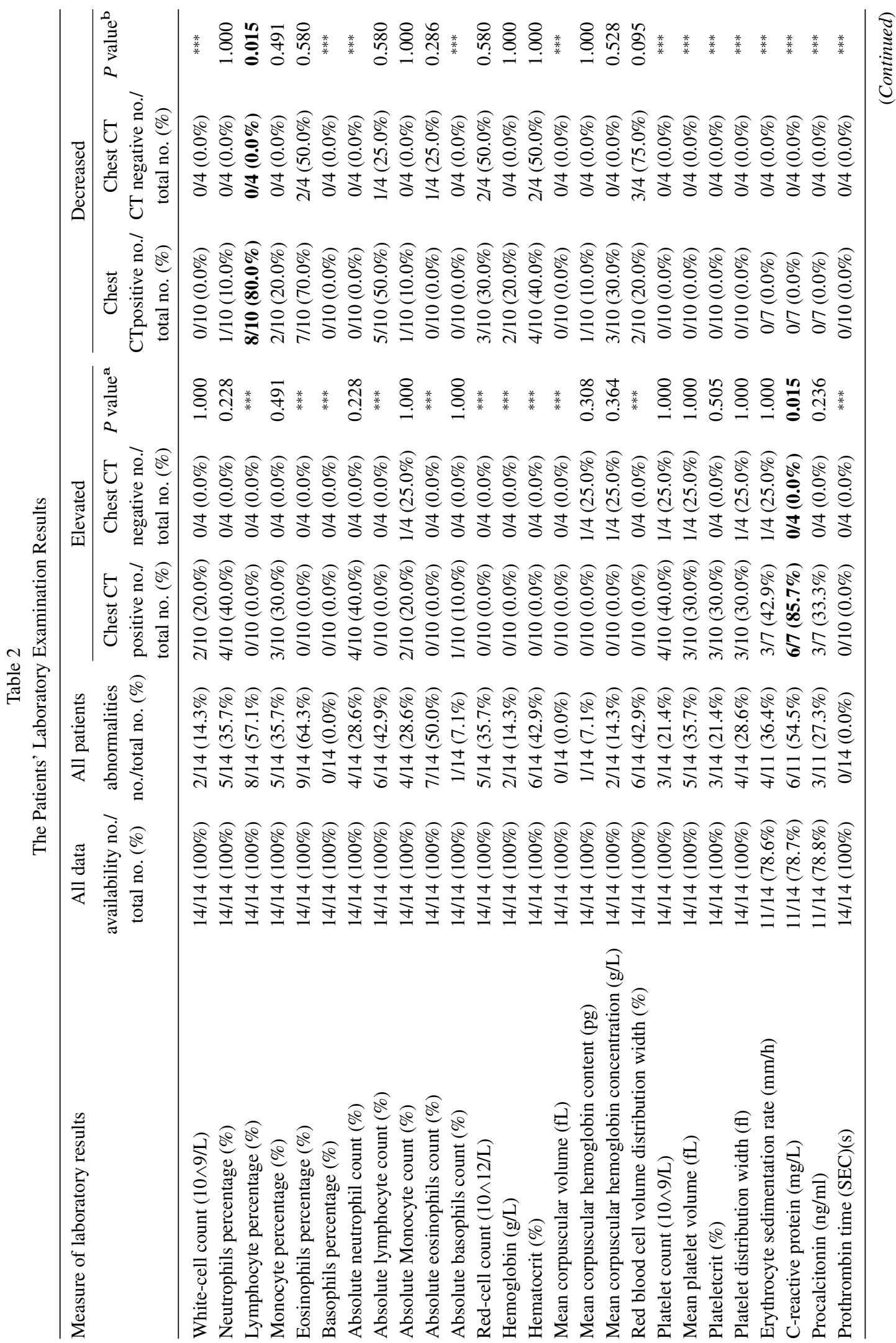




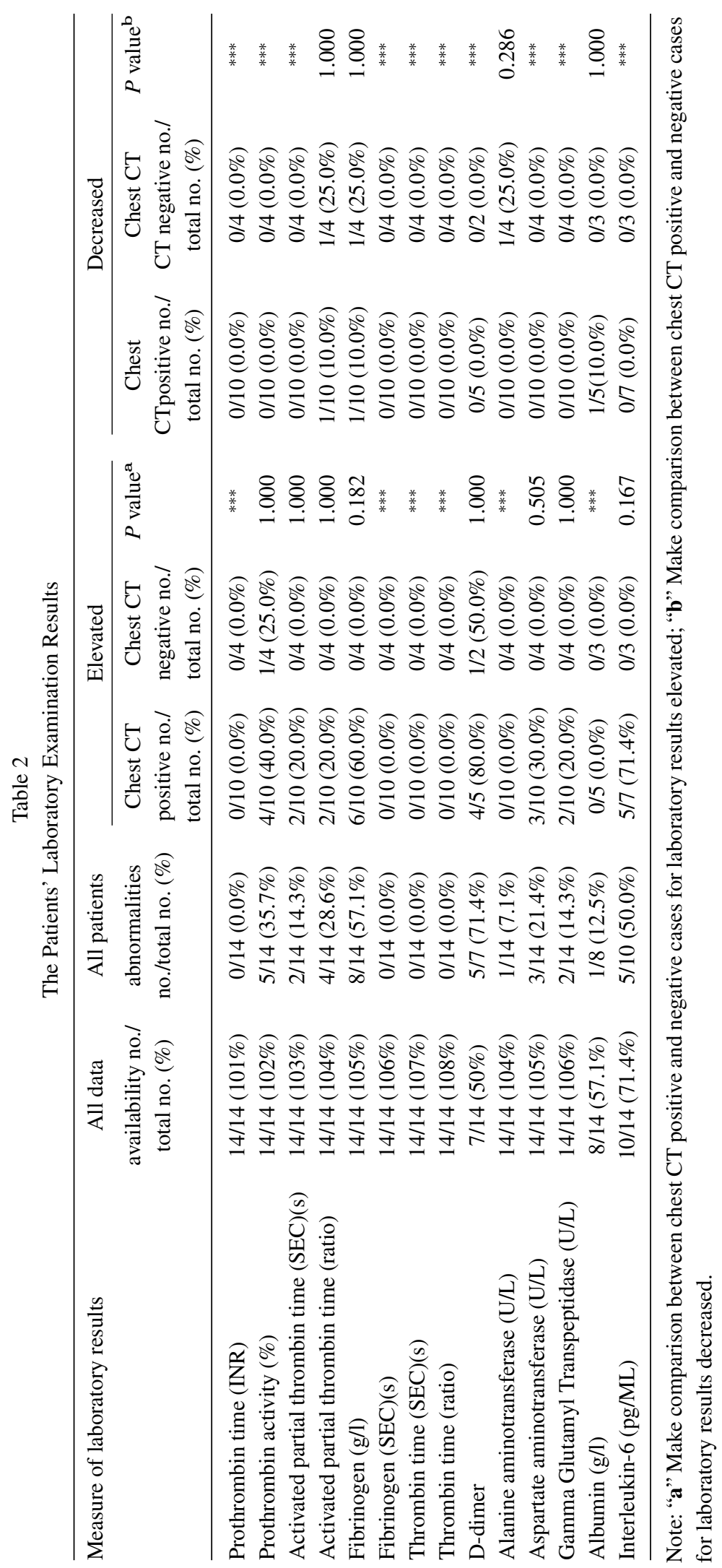




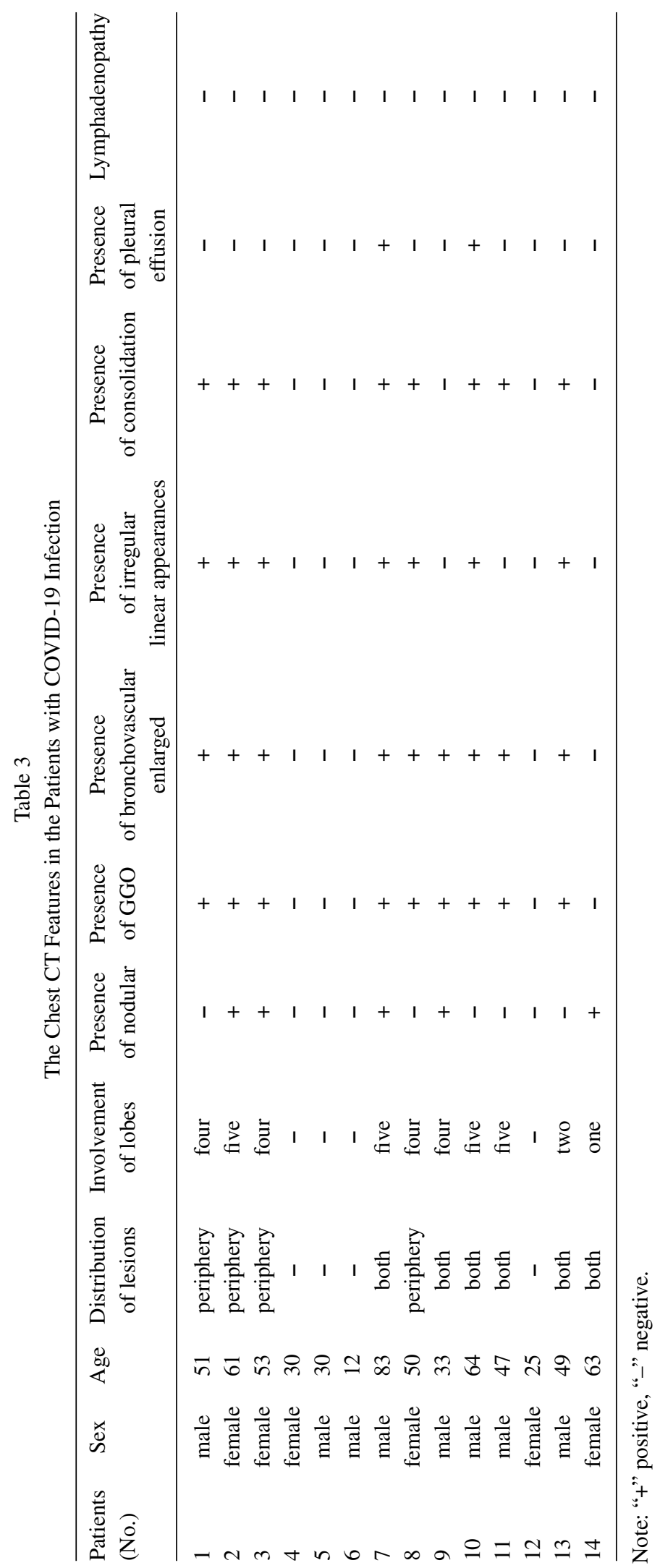




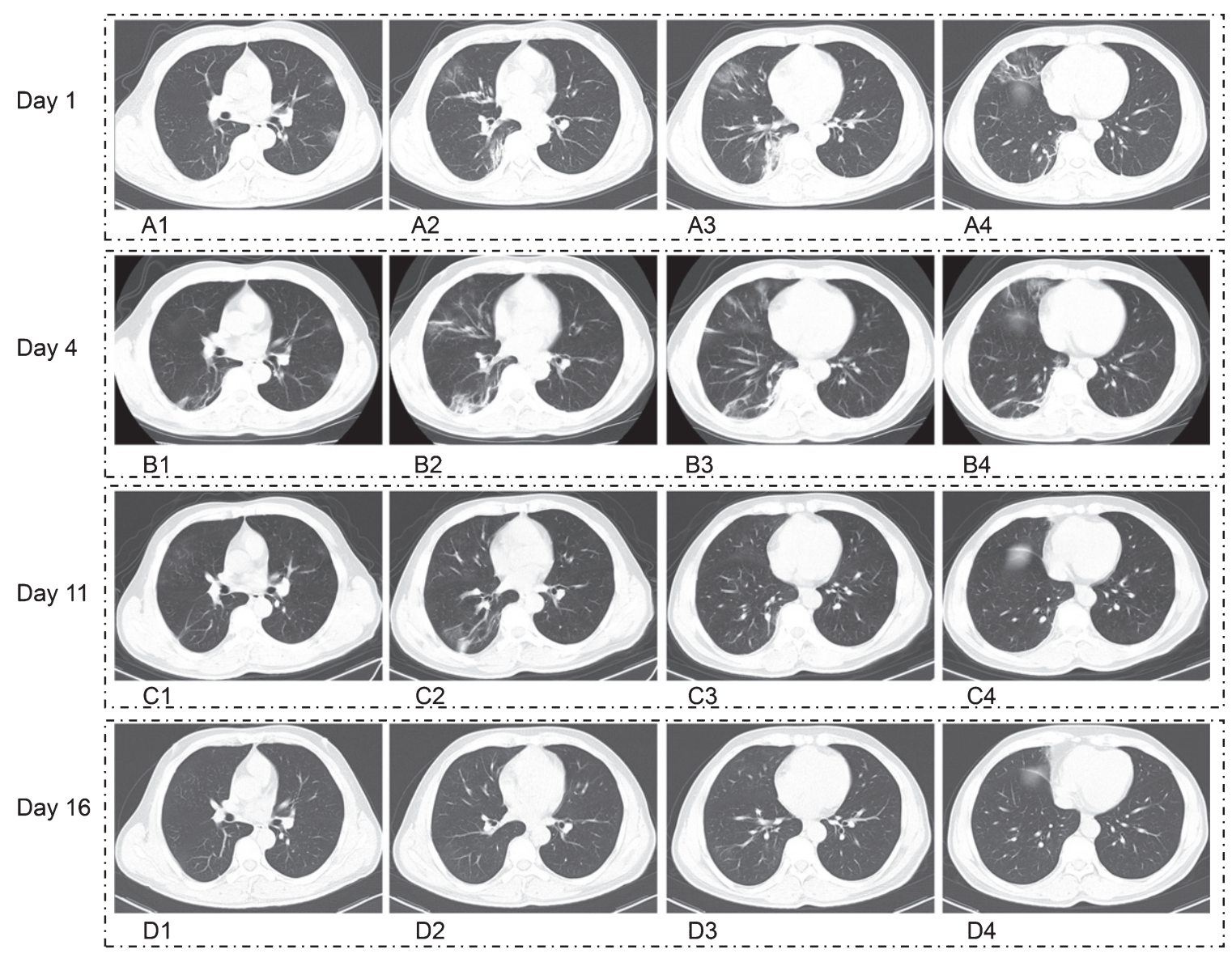

Fig. 1. Fifty-one years old man with COVID-19 pneumonia who had the history of exposure for recent travel to Wuhan, with body soreness, headache for 7 days. A1-A4. Axial computed tomographic (CT) images first time revealed that the lesions were involvement of both lung with the CT characteristics of GGO, consolidation, bronchovascular enlarged, irregular linear appearances, and most of lesions distributed peripherally, there were no the CT features of pleural effusion. B1-B4. Following up CT images on day 4 shown that lesion had been progress. C1-C4. Following up CT images on day 11 demonstrated that most of lesions had been absorption after treatment. D1-D4. Following up CT images on day 16 manifested that all of lesions had been absorption.

finding was observed in one of these two cases, and the other one with a nodular in the right lower lobe of chest CT examination.

Recently, literatures reported that GGO, consolidation, air bronchogram, and pleural effusion etc. were shown in the chest CT [6, 13], and the pleural effusion or lymphadenophathy was rare $[4,16]$. The lesions distributed in pulmonary parenchyma were single lobe $(10 / 24,42 \%)$ or bilateral multi-lobes $(10 / 24,42 \%)$ in the stage- 1 of COVID-19, single lobe $(4 / 20,20 \%)$ or bilateral multi-lobes $(16 / 20,80 \%)$ in the stage-4 [17]. In our study, the results revealed that GGO, bronchovascular enlarged in the lesions, linear appearances, and consolidations were found in the patients with COVID-19 pneumonia. The presence of pleural effusion was seen in two cases (Fig. 2). There was no case with lymphadenopathy. $(8 / 10,80 \%)$ patients with lesions involving 4 to 5 lobes distributed peripherally or subpleural regions, the most significant lesions were observed in the bilateral lower lobes, especially in the right lower lobe $(9 / 10,90 \%)$. Previous studies shown that the most frequency of lobe involvement was located in bilateral lower lobe $[16,18,19]$, especially located in the right lower lobe $[16,19,20]$. Therefore, we speculated that involvement of lower lobes (the particular involvement of right lower lobe) were the relative specific sign for the radiologists to know in the patients with COVID-19 pneumonia (Fig. 3). 


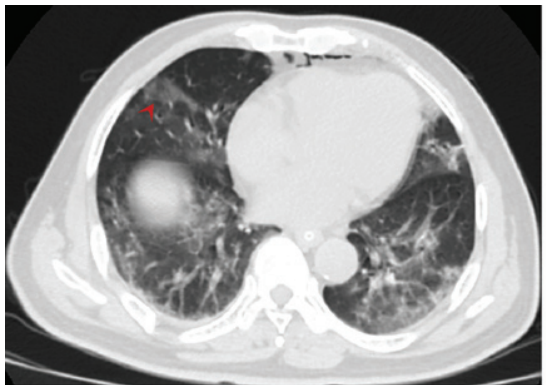

A

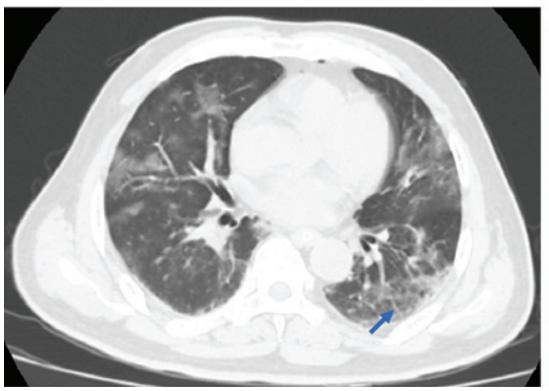

C

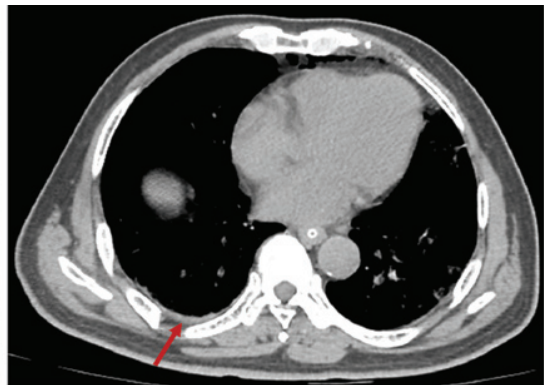

B

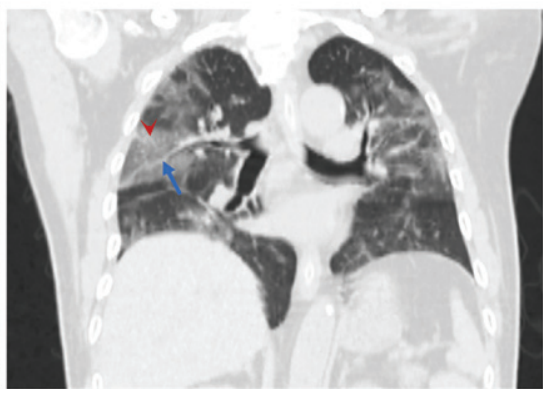

D

Fig. 2. Sixty-four years old man with COVID-19 pneumonia who had the history of exposure for recent travel to Wuhan, with fatigue for 3 days. A-C. Aixal CT images demonstrated the CT characteristics of GGO (arrow head), bronchovascular enlarged (blue arrow), and minimal pleural effusion (red arrow). Most of lesions distributed peri-bronchovascular and subpleural area. Pneumomediastinum was observed. D. Coronal reformation CT images revealed that the lesions were involvement of both lungs.

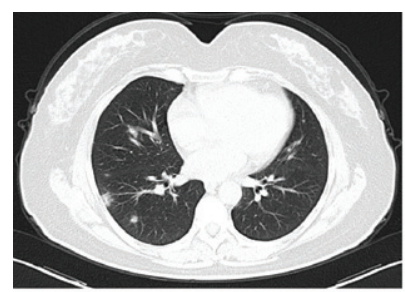

A

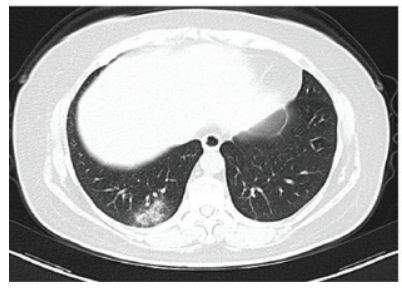

D

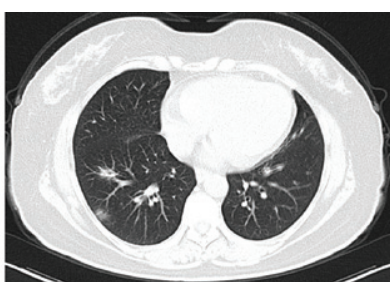

B

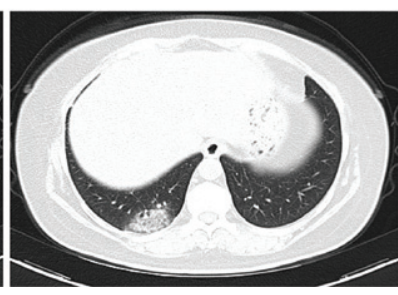

E

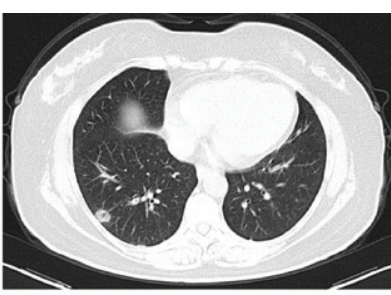

C

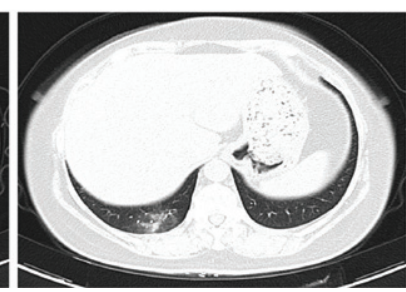

$\mathrm{F}$

Fig. 3. Fifty-three years old woman with COVID-19 pneumonia who had the history of exposure for recent travel to Wuhan, with cough for 2 days. A-F. Axial CT images shown that the ill-defined margin GGO mixed consolidation observed in right lower lobe significantly.

Generally, differential diagnosis for COVID-19 pneumonia includes virus pneumonia, fugal pneumonia, and bacterial pneumonia. The radiologic features of viral pneumonia are associated with the viral infectious pathogenesis. GGO and consolidation are the typical characteristics in most of the viral pneumonia. Not all of the viral pneumonia demonstrated with typical radiologic features because 
of the similar pathogenesis in the same viridae. However, recognizing the pneumonia patterns may aid doctor in reducing the unnecessary use of antibiotics. Therefore, the radiologic features of virus pneumonia are diverse and overlap [21]. Currently, definite diagnosis of COVID-19 infection cannot be achieved by using imaging features alone. Identification of the types of viral pathogens may not always be easy. As for the fungal pneumonia and bacterial pneumonia, consolidation plus nodules or consolidation plus GGO and nodules is suggested to diagnose for invasive fungal disease. Subsegmental or segmental consolidations are more frequent in bacterial pneumonia than in invasive fungal disease. Large nodules, as well as nodules with halo sign or both small and large nodules, are related to invasive fungal disease [22]. In addition, although rare enough, the perinodular halo is highly specific for invasive aspergillosis [23]. Therefore, identification of the types of viral pathogens may not always be easy by utilizing imaging characteristics alone. Radiologist should make diagnosis of COVID-19 by radiologic features in combination with clinical manifestation, exposure histology, and laboratory results.

The laboratory examination results suggested that the total number of white blood cells was normal in 14 patients $(12 / 14,86 \%)$. However, significant statistical differences were observed in lymphocyte percentage decreased and C-reactive protein elevated (all $P=0.015$ ), and there were no significant statistical differences in the other laboratory examination results between positive and negative chest CT results in the patients with COVID-19 infection in our study. Previous studies shown that the total number of white blood cells was normal $(590 / 978,60 \%)$, the percentage of lymphocyte count was decreased (731/879, 83\%), C-reactive protein was increased (481/793, 61\%) [24]. Therefore, Knowing the clinical information is helpful for radiologists to diagnose COVID-19 pneumonia in the patients with suspected novel coronavirus infection (2019-nCoV), and the more details of the correlation between CT findings and laboratory examinations need to be further study.

This study also has several limitations. First, the sample size was small, especially the different stages of COVID-19, we just described the clinical manifestation, laboratory examination results, and chest CT features, further research would be conduct for statistical analysis if the sample is expanded. Second, some of laboratory indexes were not available from all the study subjects in this retrospective study. Third, the more details on the correlation between CT findings and laboratory examinations need to be further study to add information for diagnosis in the patients with suspected COVID-19 infection. Last, CT features were evaluated by two experienced radiologists in consensus, inter-observer agreement for two radiologists in overall image quality or CT features evaluation was not performed.

\section{Conclusion}

This study suggested that fever and cough were most frequent in the clinical characteristics in the patients with COVID-19 infection. Lymphocyte percentage decreased and C-reactive protein elevated may provide some information of laboratory findings to diagnose COVID-19 pneumonia. MSCT is a good tool to detect early and/or mild lesions of COVID-19 pneumonia. Multiple GGO, consolidation, and bronchovascular enlarged were common seen. Pleural effusion or lymphadenopathy was rare. Furthermore, the history of exposure is also an important clue to suspect those who may be infected current novel coronavirus. Therefore, the radiologists should know the key points of clinical manifestation, laboratory findings, and exposure history to diagnose the patients with suspected COVID-19 infection in clinical practice beyond radiologic findings.

\section{Conflicts of interest}

The authors have no potential conflicts of interest to disclose. 


\section{Acknowledgments}

The authors thank Dr. Lei Wen who supported the techniques of parameters scanning for this study. The authors also gratefully thank all participants and staff of the Affiliated Hospital of Guizhou Medical University, as well as Study For Better Team who contributed their best research spirits to each other during the process of the study.

\section{References}

[1] Q. Li, X. Guan and P. Wu, et al, Early transmission dynamics in Wuhan, China, of novel coronavirus-infection pneumonia, $N$ Engl J Med, published online Jan. 29, 2020. DOI: 10.1056/NEJMoa2001316.

[2] R.W. Barrette, S.A. Metwally and J.M. Rowland, et al, Discovery of swine as a host for the Reston ebolavirus, Science 325(5973) (2009), 204-206

[3] S.A. Rasmussen and D.J. Jamieson, 2009 H1N1 influenza and pregnancy - 5 years later, N Engl J Med 371(15) (2014), 1373-1375

[4] World Health Organization (WHO), Coronavirus disease (COVID-2019) situation reports-36 [EB/OL], https:// www.who.int/docs/default-source/coronaviruse/situation-reports/20200225-sitrep-36-covid-19.pdf?sfvrsn=2791b4e0 -2, 2020-02-25.

[5] Song F, Shi N, Shan F, et al, Emerging Coronavirus 2019-nCoV Pneumonia, Radiology, published online Feb. 06, 2020. DOI: 10.1148/radiol.2020200274.

[6] Shi H, Han X, Zheng C, Evolution of CT Manifestations in a Patient Recovered from 2019 Novel Coronavirus (2019nCoV) Pneumonia in Wuhan, China, Radiology, published online Feb. 07, 2020. DOI: 10.1148/radiol.2020200269.

[7] Fang Y, Zhang H, Xu Y, et al., CT manifestations of two cases of 2019 novel coronavirus (2019-nCoV) pneumonia, Radiology, published online Feb. 07, 2020. DOI: 10.1148/radiol.2020200280.

[8] Paul NS, Roberts H, Butany J, et al, Radiologic pattern of disease in patients with severe acute respiratory syndrome: the Toronto experience, Radiographics 24(2) (2004), 553-563

[9] Huang P, Liu T, Huang L, et al, Use of chest CT in combination with negative RT-PCR assay for the 2019 novel coronavirus but high clinical suspicion, Radiology, published online Feb. 12, 2020. DOI: 10.1148/radiol.2020200330.

[10] Phan LT, Nguyen TV, Luong QC, et al, Importation and human-to-human transmission of a novel coronavirus in vietnam, N Engl J Med, published online Jan. 28, 2020. DOI: 10.1056/NEJMc2001272.

[11] Holshue ML, DeBolt C, Lindquist S, et al, First case of 2019 novel coronavirus in the United States, $N$ Engl J Med, published online Jan. 31, 2020. DOI: 10.1056/NEJMoa2001191.

[12] Xie X, Zhong Z, Zhao W, et al., Chest CT for typical 2019-nCoV pneumonia: relationship to negative RT-PCR testing, Radiology, published online Feb. 12, 2020. DOI: 10.1148/radiol.2020200343. [Epub ahead of print]

[13] Kanne JP. Chest CT findings in 2019 novel coronavirus (2019-nCoV) infections from Wuhan, China: Key points for the radiologist, Radiology, published online Feb. 04, 2020. DOI: 10.1148/radiol.2020200241.

[14] Lei J, Li J, Li X, Qi X, CT imaging of the 2019 novel coronavirus (2019-nCoV) pneumonia, Radiology, published online Jan. 31, 2020. DOI: 10.1148/radiol.2020200236.

[15] Huang C, Wang Y, Li X, et al, Clinical features of patients infection with 2019 novel coronavirus in Wuhan, China, Lancet, 395 (2020), 497-506

[16] Chen N, Zhou M, Dong X, et al, Epidemiological and clinical characteristics of 99 cases of 2019 novel coronavirus pneumonia in Wuhan, China: a descriptive study, Lancet, 395 (2020), 507-513

[17] Bernheim A, Mei X, Huang M, et al, Chest CT findings in coronavirus disease-19 (COVID-19): relationship to duration of infection, Radiology, published online Feb. 20, 2020. DOI: 10.1148/radiol.2020200463.

[18] Pan F, Ye T, Sun P, et al, Time course of lung changes on chest CT during recovery from 2019 novel coronavirus (COVID-19) pneumonia, Radiology, published online Feb. 13, 2020. DOI: 10.1148/radiol.2020200370.

[19] Liu P, Tan XZ, 2019 novel coronavirus (2019-nCoV) pneumonia, Radiology, published online Feb. 04, 2020. DOI: 10.1148/radiol.2020200257.

[20] Chung M, Bernheim A, Mei X, et al, CT imaging features of 2019 novel coronavirus (2019-nCoV), Radiology, published online Feb. 04, 2020. DOI: 10.1148/radiol.2020200230.

[21] Koo HJ, Lim S, Choe J, et al, Radiographic and CT features of viral pneumonia, Radiographics, 38 (2018), 719-739 
[22] Chen W, Xiong X, Xie B, et al, Pulmonary invasive fungal disease and bacterial pneumonia: a comparative study with high-resolution CT, Am J Transl Res, 11 (2019), 4542-4551

[23] Bruno C, Minniti S, Vassanelli A, Pozzi-Mucelli R, Comparison of CT features of Aspergillus and bacterial pneumonia in severely neutropenic patients, J Thorac Imaging, 22 (2007), 160-165

[24] Guan WJ, Ni ZY, Hu Y, et al, Clinical characteristics of coronavirus disease 2019 in China, $N$ Engl J Med, published online Feb. 28, 2020. DOI:10.1056/NEJMoa2002032 